\title{
Heel blood sampling in preterm infants: which technique?
}

\author{
D P Barker, B W Latty, N Rutter
}

\begin{abstract}
Preterm infants undergoing heel blood sampling were randomly allocated to specimen collection by heel puncture (Autolet II Clinisafe) or incision (Tenderfoot 'preemie').

A total of 187 procedures was observed in 47 infants. No significant difference was found in the infants' behavioural response, increase in heart rate, or in the frequency of specimen haemolysis. Collection times for small to medium sized samples were similar, but for large samples $(>1 \mathrm{ml})$ the Tenderfoot method was superior and fewer repeat procedures were necessary. Tenderfoot has the added advantage of improved safety, but it is too costly for routine use. (Arch Dis Child 1994; 71: F206-F208)
\end{abstract}

Heel prick is the most common method of obtaining blood samples in newborn infants. It is performed routinely for biochemical screening and many infants have additional samples collected for bilirubin and glucose measurement. Sick infants receiving intensive care often undergo repeated heel pricks.

Heel puncture with a lancet is the standard method for obtaining the sample. This can be performed manually, but spring loaded devices are equally effective and cause less distress. ${ }^{1} \mathrm{~A}$ new device using a different technique is now available. It produces a small skin incision of defined length and depth from a spring loaded surgical blade. The device, called Tenderfoot (International Technidyne Corporation), is a sterile, self contained, single use unit, which has several theoretical advantages for specimen collection. The controlled incision depth is designed to reach superficial dermal blood vessels while avoiding deeper dermal pain fibres. Increased blood flow from the cut may reduce the sample collection time, with additional benefits of less pain, heel trauma, and sample haemolysis from squeezing.

We carried out a randomised trial comparing this device with a spring loaded lancet, the Autolet II Clinisafe (Owen Mumford), which we have used routinely for several years.

Department of

Neonatal Medicine and

Surgery, Nottingham

City Hospital,

Hucknall Road

Nottingham NG5 1PB

D P Barker

B W Latty

N Rutter

Correspondence to:

Dr Barker.

Accepted 21 June 1994 ment. Written parental consent was obtained and the study was approved by the ethical committees of the two hospitals.

\section{Method}

The study was conducted over a four the neonatal intensive care Nottingham City and University Hospitals. The subjects were preterm infants requiring
SUBJECTS

Forty seven infants, with gestational ages from 23 to 37 weeks (median 31 weeks) and birth weights from 560 to $3220 \mathrm{~g}$ (median $1720 \mathrm{~g}$ ) were enrolled into the study. Weight at entry varied from 740 to $3520 \mathrm{~g}$ (median $1720 \mathrm{~g}$ ).

\section{PROTOCOL}

Blood sampling was performed by one of eight experienced neonatal senior house officers, after instruction in the use of the Tenderfoot device. Most specimens were taken before the morning ward round, with the remainder collected during normal working hours. All procedures were observed by a research fellow (DPB) and a staff nurse (BWL).

Before the infant was disturbed, baseline heart rate (where monitored) and behavioural state were noted. Infants were then randomly allocated to have blood taken using either the Tenderfoot 'preemie' (incision depth 0.85 $\mathrm{mm}$, length $1.75 \mathrm{~mm}$ ) or Autolet II Clinisafe with neonatal lancet and platform (puncture depth $2.16 \mathrm{~mm}$ ). Randomisation was stratified into three groups $(<1000 \mathrm{~g}, 1000-1500 \mathrm{~g}$, $>1500 \mathrm{~g}$ ) according to the infant's latest weight.

In the first five seconds after firing of the device the infant's facial response was closely observed. The presence or absence of cry was noted, along with any changes in facial expression (eye squeeze and deepening of the nasolabial furrow), which typify neonatal reactions to brief invasive events. ${ }^{2}$ The maximum heart rate reached during the procedure, the length of the procedure, the number of repeats necessary to complete specimen collection, and the nature of the specimen were also recorded. Specimens sent to the clinical chemistry laboratory were examined for haemolysis without indication of which device had been used.

To allow comparison between devices the amount of blood collected on each occasion was allocated a score from 1 to 4 . Thus the smallest amount of blood (one or two capillary tubes for blood gas or bilirubin measurement) was assigned a score of 1 . A specimen of volume sufficient for a full blood count or urea and electrolytes was assigned a score of 2 . Collection of a moderate amount (for example, full blood count plus urea and electrolytes) was assigned a score of 3 , and any additional investigations (for example, full blood count, urea and electrolytes, plus theophylline or gentamicin concentration) converted this to a score of 4. The actual volumes of blood encompassed by this scoring system were estimated to vary from 0.1 to $1.5 \mathrm{ml}$. 


\section{Results}

One hundred and eighty seven heel blood sampling procedures were observed, 97 with the Autolet and 90 with the Tenderfoot device. Most infants (26/47, 55\%) were included in the study on only one or two occasions. Thirteen $(28 \%)$ were included between three and seven times and eight were included more than seven times. The median sample score for all specimens, and for those taken using the two devices separately, was 3 .

\section{COLLECTION TIME}

The collection times for different sample sizes were compared (see table). For small to medium samples (sizes 1-3) there was no significant difference between the devices. For large samples (size 4) the median collection time was reduced by almost one and a half minutes using the Tenderfoot device $(p<0.05$, Mann-Whitney U test).

NUMBER OF REPEAT PROCEDURES REQUIRED Repeat heel pricks were necessary on 40 of 97 occasions (41\%) with the Autolet, compared with 17 of 90 occasions $(19 \%)$ with the Tenderfoot $\left(p=<0.005, \chi^{2}\right.$ test). More than one repeat (maximum four repeats) was required on 12 occasions with the Autolet, but only once with the Tenderfoot device. With both devices, over three quarters of repeats occurred during collection of sample sizes 3 or 4 . The total number of procedures performed was 154 with the Autolet and 108 with the Tenderfoot device.

\section{BEHAVIOURAL RESPONSE}

Facial expression and cry in the first five seconds after firing of the device were analysed, excluding 17 occasions (among five infants) where ventilation or systemic analgesia were in progress. This group also included the one infant with serious intracranial pathology (grade 3 intraventricular haemorrhage). From 87 heel blood sampling procedures with the Autolet no response was observed on 32 occasions $(37 \%)$, compared with 33 of 83 occasions $(40 \%)$ with the Tenderfoot. Thus most infants reacted with facial movements with or without cry, suggesting pain perception. In the Autolet group crying with eye squeeze and deepening of the nasolabial fold was the most consistent response (31/55, $56 \%$ ), with the remainder showing a change in facial expression without cry. In the Tenderfoot group these proportions were reversed, with change in facial expression but no cry as the most frequent response $(28 / 50$,

Sample collection times

\begin{tabular}{|c|c|c|c|}
\hline \multirow[b]{2}{*}{ Sample } & \multicolumn{2}{|c|}{ Median (interquartile range) sampling time (min) } & \multirow{2}{*}{$\begin{array}{l}\text { Significance } \\
\text { (Mann-Whitney } \\
\text { U test) }\end{array}$} \\
\hline & Autolet & Tenderfoot & \\
\hline $\begin{array}{l}\text { Size } 1(n=42) \\
\text { Size } 2(n=43) \\
\text { Size } 3(n=45) \\
\text { Size } 4(n=57)\end{array}$ & $\begin{array}{l}0.81(0.55-1.05) \\
2.50(1.98-2.43) \\
5.38(3.45-7.32) \\
6.43(5.00-8.37)\end{array}$ & $\begin{array}{l}0.63(0.47-0.92) \\
2.40(1.85-3.78) \\
4.92(3.08-5.87) \\
5.00(4.10-6.43)\end{array}$ & $\begin{array}{l}\text { NS } \\
\text { NS } \\
\text { p }<0.05\end{array}$ \\
\hline
\end{tabular}

$56 \%$ ), although this difference was not statistically significant.

\section{HEART RATE}

Heart rate was monitored as part of clinical management on $81 \%$ of occasions. Subtraction of the baseline from the maximum value allowed calculation of the change in heart rate. There was a median rise of 27 beats/minute, with no difference between the devices.

\section{HAEMOLYSIS}

One hundred and twenty one of 187 specimens $(65 \%)$ were examined for haemolysis by the department of clinical chemistry. Haemolysis was present in 17 of $61(28 \%)$ specimens obtained with the Autolet compared with 12 of $60(20 \%)$ obtained with the Tenderfoot. This difference was not statistically significant.

\section{Discussion}

Blood sampling from the heel is the most common invasive procedure performed on newborn infants. Although the trauma caused by individual heel pricks is not great, some preterm infants undergo over 200 such procedures during their stay on the neonatal unit. $^{3}$ The behavioural and physiological responses of preterm and term infants to heel prick have been well documented. ${ }^{45}$ There is evidence of hypersensitivity with repeated heel trauma ${ }^{6}$ Measures proposed to lessen the pain of heel prick include the use of mechanical lancets instead of manual heel prick, ${ }^{1}$ and the application of topical local anaesthetic. ${ }^{7}$ In addition to pain and distress, repeated heel pricks predispose to the development of calcaneal osteomyelitis, which can disturb hind foot growth, and implantation epidermoid cysts. ${ }^{89}$ The risks of these can be minimised by attention to technique and asepsis. ${ }^{10}$

Blood sampling from the heel is also time consuming, with junior medical staff spending up to two hours each morning on a blood round'. In this study doctors spent an average of four minutes with each infant on sample collection. Handwashing, assembly of the Autolet and preparation of the heel is likely to at least double this figure. A device which reduces specimen collection time without increasing pain would therefore be beneficial.

A study in term infants comparing the Tenderfoot with a spring loaded lancet (Microtainer, Becton Dickinson) found substantial reductions in specimen collection time. ${ }^{11}$ In this study we have shown that the Autolet II Clinisafe and Tenderfoot 'preemie' perform equally well for the collection of small to medium sized blood samples from the heels of preterm infants. For large specimens $(>1 \mathrm{ml})$ the Tenderfoot is more effective than the Autolet. In addition to the shorter collection time, the number of occasions on which repeat procedures were necessary was reduced by half, and of these more than one repeat was rarely needed using the Tenderfoot. 
In around $60 \%$ of cases behavioural observation suggested pain perception, with little difference between devices. We could thus not substantiate the manufacturer's claim that the Tenderfoot 'virtually eliminates' pain. As there were fewer repeats with the Tenderfoot, however, the pain of the procedure was indirectly reduced.

Other benefits of the Tenderfoot were noted. Unlike the Autolet II Clinisafe it does not require assembly, and after single use is immediately discarded. This minimises the risk of needle stick injury, and also that of cross infection, which has been reported with the Autolet. ${ }^{12}$ Examination of the heel 24 hours after using the Tenderfoot revealed in most cases an incision mark but no bruising. This was not studied systematically, but it was our impression that apart from fewer repeat sampling sites the heels generally appeared less traumatised after Tenderfoot use.

The Tenderfoot does, however, have certain disadvantages. Unlike the Autolet the precise sampling point on the heel is not visible at the time of firing. It is therefore more difficult to direct, and in the smallest infants, in whom heel width may be equal or less than that of the base of the device, this may result in the incision being made in a suboptimal area. The other main drawback is cost. Based on a survey of invasive procedures from our own neonatal unit, we estimate that around 8000 heel pricks are performed each year. At current prices the cost implication of using a Tenderfoot device for each of these, with a repeat rate of $19 \%$ as in this study, would amount to $£ 7700$. By comparison, the cost of equipping a 30 cot neonatal unit with Autolet II Clinisafe devices and providing 8000 neonatal lancets and platforms (which are reusable during individual procedures) is around one tenth of this figure.

In conclusion, for the collection of small to medium samples the Autolet and Tenderfoot perform equally well. Where a large sample volume is needed the Tenderfoot reduces collection time and the need for repeats. It has the added advantage of improved safety, but is too costly for routine use.

The authors thank the International Technidyne Corporation for supplying the Tenderfoot devices used in this study.

1 Harpin V, Rutter N. Making heel pricks less painful. Arch Dis Child 1983; 58: 226-8

2 Grunau RVE, Johnston CC, Craig KD. Neonatal facial and ry responses to invasive and non-invasive procedures. Pain 1990; 42: 295-305.

3 Barker DP, Rutter N. Exposure to invasive procedures during neonatal intensive care unit admissions. Arch Dis Child (in press).

4 McIntosh N, Van Veen L, Brameyer H. The pain of hee prick and its measurement in preterm infants. Pain 1993; 52: $71-4$.

5 Craig KD, Whitfield MF, Grunau RVE, Linton J Hadjistavropoulos HD. Pain in the preterm neonate: behavioural and physiological indices. Pain 1993; 52 287-99.

6 Fitzgerald M, Millard C, McIntosh N. Cutaneous hypersensitivity following peripheral tissue damage in newborn infants and its reversal with topical anaesthesia. Pain 1989; 39: 31-6.

7 Wester U. Analgesic effect of lidocaine ointment on intact skin in neonates. Acta Paediatr 1993; 82: 791.

8 Borris LC, Helleland H. Growth disturbance of the hind part of the foot following osteomyelitis of the calcaneus in the newborn. F Bone foint Surg 1986; 68: 302-5.

9 Cartlidge PHT, Fox PE, Rutter N. The scars of newborn intensive care. Early Hum Dev 1990; 21: 1-10.
int

10 Blumenfeld TA, Turi GK, Blanc WA. Recommended site and depth of newborn heel skin punctures based on anatomical measurements and histopathology. Lancet 1979; i: 230-3.

11 Matthews D. Clinical considerations for infant heel blood sampling: comparative studies of time requirement and repeat sticks during heelstick. Neonatal Intensive Care 1992; May/June: 66-8.

12 Polish LB, Shapiro CN, Bauer F, et al. Nosocomial transmission of hepatitis $B$ virus associated with the use of a spring-loaded finger stick device. $N$ Engl f Med 1992; 326: 721-5. 